\title{
Exact number representations in first and second language
}

One of the major questions in the field of cognitive psychology is the extent to which our thought is dependent on, or formed by, the language we speak. In the mid-1900s, proponents of the linguistic relativity principle claimed that different languages with distinct grammatical properties and lexicons would have a major impact on the way the native speakers of that language perceived reality. This idea was based on the work of the anthropologists Sapir (1949), and Whorf (1956), and named the "Sapir-Whorf-Hypothesis" by Hoijer (1971). The opposite view is expressed by the theory of cultural universality ( $\mathrm{Au}, 1983$ ), meaning that basic concepts innate to human beings can be found in every culture irrespective of linguistic differences.

The concept of number seems to be a good example for a theory of cultural universality at first sight, as all known cultures have developed at least some number words, and even pre-verbal infants and animals are able to single out the larger of two sets based on the respective number of items. The term "numerosity" was used by Dehaene (1997) for the awareness of quantity. Yet, it is still not clear whether nature has provided us with the concept of exact number or if this is a cultural acquirement based on the acquisition of verbal counting procedures.

This chapter will review evidence supporting the language relativity hypothesis for the instance of exact number representations in a small number range (up to 10); other chapters in this book focus on the linguistic specificities of multi-digit number word systems and other aspects of mathematics Bahnmüller, this volume; Dowker, this volume). Presenting studies from different fields, this chapter will propose that the concept of exact numerosity is based on natural language, and furthermore that linguistic specificities even put constraints on the form of exact numerosity representations. The first focus is on the finding that grammatical properties shape the development of the concepts for one versus two, three, and more. Second, studies that describe a representational change in adults who learn a new number word system (including symbols for numerosities higher than four or five) will be presented. Third, differences in arithmetic fact retrieval in both first and second language will be reviewed. These findings will be discussed in the light of the "access-deficit-hypothesis" regarding developmental dyscalculia, suggesting that children with mathematical difficulties may have a problem in accessing number magnitude from symbols (e.g., presenting with longer response times 
or less matured reaction time patterns) rather than in processing quantities per se (Rousselle \& Noël 2007). The chapter concludes with the proposal that using canonical finger configurations provide a language-independent means of symbolizing exact quantities for children presenting with problems in this area.

\section{The role of language for the ontogenetic development of exact numerosity concepts in early childhood}

The mapping of number words to their corresponding numerosities develops gradually: First, children understand the cardinality meaning of the number word "one," then they differentiate "two" from other number words, next comes "three," and at some point the relationship between counting and numerosity is understood and the cardinality principle generalizes to all number words in the counting sequence. Wynn (1990, 1992) named these levels 0-knowers, 1-knowers, 2-knowers, 3-knowers, and cardinality-principle-knowers. According to Wynn (1990), children acquire the last stage at an approximate age of 3.5 years and use counting to refer the numerosities of sets only from this stage onward. She argued that mapping number words and their respective numerosities is a kind of associative learning process which is possible because children already know that number words correspond to specific, unique, and inherently ordered numerosities, even before they map each word to its numerosity (Wynn, 1992). The reason why children learn to map smaller number words to their numerosity first would be the higher frequency of smaller numbers in the children's environment (Wynn, 1990). However, there may also be language-related aspects at play. The scientific controversy regarding the role of language for the first developmental steps toward an exact number representation is reviewed in the next sections.

\subsection{The language-irrelevant hypothesis}

According to the language-irrelevant hypothesis, the dominant role in acquiring the concept of cardinality should lie in recognizing the equivalence of numerosities of sets via one-to-one correspondence. As first prominent proponents of this view, Gelman and Gallistel (1978) stated that preschool children already understand the principles underlying counting due to their correspondence to this pre-verbal numerosity coding mechanism, and young children's failure to execute 
the counting process successfully would occur only because of high performance demands (Greeno et al., 1984; but see Le Corre et al., 2006 for opposite results). According to this view, mapping number words and their respective numerosities is just a kind of associative learning. The reason why children learn to map smaller number words first should be the higher frequency of smaller numbers in the children's environment. Butterworth (2010) also postulated that pre-counting children and animals possess an ordered sequence of numerosity concepts (numerosity coding). Furthermore, in this pre-verbal numerosity coding it is assumed that the individual is able to establish numerical equivalence of two sets through oneto-one correspondence. He emphasized this view with the following statement: "The concept of fiveness pre-exists acquisition of the knowledge that the word five refers to the numerosity fiveness" (p. 538).

\subsection{The strong language hypothesis}

The perspective opposing the language-irrelevant hypothesis is held by the strong language hypothesis (see Brannon \& Van de Walle, 2001 for an overview of this controversy). This strong language view is characterized by the assumption that the concepts of exact number and even abstract numerosity can develop only as a consequence of cardinality understanding, which in turn has been shown to result from acquiring the verbal counting process (Wynn, 1990, 1992). For example, Brannon and Van de Walle (2001) showed that 2-year-old children could not make successful ordinality judgments (determining which of two sets contains more items after being asked which one was "the winner") as long as they had not acquired any number word knowledge.

Most researchers in favor of the strong language hypothesis argue that for success in their tasks, children have to explicitly draw their attention to the numerosity of the stimuli. Furthermore, they postulate that number becomes a salient dimension of the environment to which children will consciously attend only after they have developed at least some cardinality understanding (Brannon \& van der Walle, 2001; Rousselle et al., 2004).

\subsection{The weak language hypothesis}

An intermediate view within this debate is represented by the weak language hypothesis (Brannon \& Van de Walle, 2001). The most important representative of the weak language hypothesis is the bootstrapping theory (Carey, 2001; Spelke \& Tsivkin, 2001; see next paragraph). Similarly to the strong language hypothesis, 
this theory states that children are not able to represent large exact numerosities before they have acquired the cardinality meaning of numbers (Sarnecka \& Carey, 2008).

"Bootstrapping" describes the processes by which children learn the mapping of number words to their corresponding numerosities (Wynn, 1990, 1992) building on the following cognitive prerequisites: (i) the ability to attentively track up to three objects in parallel via representing each item with a separate symbol (object file tracking system or OTS), (ii) the ability to learn meaningless ordered word lists (like hickory-dickory-dock or one-two-three-four), and (iii) the ability to understand quantity markers in language such as the singular/ plural distinction. The role of quantity markers will specifically be reviewed in Section 1.4 .

The most important difference between the strong and the weak language hypotheses is that according to the bootstrapping view, children can represent small numerosities up to three exactly before they have learned how to count.

Evidence for the weak and strong language hypotheses comes from studies showing that in some tasks toddlers don't seem to know that number words refer to specific numerosities (Condry \& Spelke, 2008; Sarnecka \& Gelman, 2004), which would not be expected by the language-irrelevant hypothesis. More specifically, Sarnecka and Gelman (2004) showed that children without full cardinality understanding did not know that equal sets must have the same number word, although they judged that the application of unmapped number words changes when numerosity changes (by adding or taking away an object of a set). Furthermore, Condry and Spelke (2008) found that young children did not know that a large unmapped number word (like "eight") continues to apply to a set whose members are rearranged or that a specific number word ceases to apply if the set is increased by one, doubled, or halved.

\subsection{The role of grammar for the development of cardinality understanding}

The bootstrapping theory explicitly states that the ability to understand singular/plural markers is a prerequisite for the mapping of number words to their corresponding numerosities (see above).

Koudier et al. (2006) investigated the developmental sequence of singular/ plural distinctions in 20- to 36-month-old infants using a preferential-looking paradigm. In their experiment, children were presented with sets containing either one item or more than one item concurrent to hearing different sentences asking them to look: These sentences marked number either only with 
noun morphology ("Look at the blicketS"/“Look at the blicket”) or redundantly with noun morphology, lexical quantifiers, and verb morphology ("Look, there ARE SOME blicketS”/“Look, there IS A blicket”; Koudier et al., 2006: (1). What they found was that 20-month-old infants were not able to look at the set corresponding to the sentence, 24-month-old children looked at the corresponding set only in the redundant sentence condition, and the 36-month-olds succeeded even in the noun morphology only condition. The authors concluded that "infants first come to understand the semantic force of the singular/plural distinction in the months just before their 2nd birthday" (Koudier et al., 2006: 2).

Similarly, Barner et al. (2007) could show that children up to the age of 20 months failed not only to comprehend singular-plural morpho-syntactic cues in a manual search task, but did not even distinguish sets of 1 from 4, although they were able to do so within the limit of object-based attention (e.g., 1 vs. 3). By the age of 22 months, some children were able to distinguish 1 from 4 in both verbal and nonverbal trials. Most interestingly, success in the manual search task in the age group of 22-24 months was due to the children who were beginning to produce plural nouns according to parental reports, indicating that the linguistic and conceptual abilities became available at around the same time.

These studies confirm that a specific grammatical aspect, namely, the number of different plural markers used within a sentence, and the individual ability to understand and use plural markers, play a role in the development of exact number representations. Interestingly, this effect can be found among children of different ages within one language community, and also in crosslanguage comparison studies.

Languages differ in the number of plural markers, with some using hardly any - like Japanese. Sarnecka et al. (2007) compared children learning English, Russian (which uses plural markers), and Japanese within the age range of 2-1/2 to 3-1/2 years to match the one from Wynn's (1990) study. Children were asked to complete counting tasks and a task where children are required to give a specific amount when asked, also known as a Give-N task. Their main finding was that more English and Russian learners than Japanese learners were 1-knowers, 2-knowers, 3-knowers, and cardinality-principle-knowers. Their interpretation was that the learning of the exact number concepts of one, two, and three is rather supported by the conceptual framework of grammatical number than implicit understanding of integers.

These developmental studies (Koudier et al., 2006; Barner et al., 2007; Sarnecka et al., 2007) strongly support the notion of linguistic constraints on the development of numerical concepts. On the other hand, the object file tracking system, which allows us and even pre- and nonverbal groups to individuate up to 
three in most children or four in most adults objects in parallel, seems to have posed constraints on the development of grammatical aspects regarding number morphology. Franzon et al. (2019) compared the number morphology of 218 different languages. The possibilities of morphological expressions for number values included few (paucal), two (dual), three (trial), and possibly sometimes even four (quadral) options. They concluded that nonverbal numerical cognition, specifically the object tracking system, constitutes a core part of language when it comes to numerical expressions. These two notions may seem contradictory at first sight, posing a "chicken-and-egg-problem": How can numerical concepts, which seemingly rely on language properties for their individual development, have constrained the cultural development of the same language properties? However, one should take into consideration that the mechanisms underlying ontogenetic and phylogenetic (including cultural) development need not be identical.

The next section will focus on observations obtained in adults whose first language contains no words for numerosities larger than four or five, investigations of their restricted numerical concepts, and the effect of learning a more elaborate number word system in a second language. These studies can be seen as providing crucial evidence for the language dependency of number concepts, even if the results of the developmental studies presented so far may not be conclusive.

\section{The impact of number word systems on exact number representations}

Strong evidence for language determining thought at least in one instance comes from observations in two independent Amazonian cultures with very restricted counting systems, namely, the Pirahã (Gordon, 2004) and the Mundurukú (Dehaene et al., 2008; Pica et al., 2004), and from deaf individuals who do not have access to a usable model for spoken or signed language, but live in a numerate culture (Spaepen et al., 2010).

Members of the Pirahã tribe use only number words for one, two, and many (Gordon, 2004). In informal observations they show no recursive use of their restricted count system, which means that they do not combine these words to depict larger quantities. They use fingers to supplement oral enumeration, but inaccurately even for numbers smaller than five. Furthermore, the word for one is sometimes also used to denote small quantities such as two or three, appearing to mean "roughly one." More formal examinations revealed that their 
restricted number word system limits their ability to exactly enumerate sets exceeding two or three items. For tasks requiring additional cognitive processing, their performance deteriorated even in this small number range. Gordon's (2004) interpretation of these results was that the Pirahã used magnitude estimation to solve the tasks, meaning that they did not use counting even in the smallest number range. He concluded that humans who are not exposed to a number word system cannot represent exact quantities even for medium-sized sets of four or five.

The Mundurukú lack number words for quantities beyond five, but are able to compare and add large quantities far larger than their naming range (Pica et al., 2004). However, they fail in exact arithmetic tasks using numbers larger than four or five. The authors come to the same conclusion as Gordon (2004), namely, that language plays a special role in the emergence of exact number representations during development. They also state that the availability of number names may not be sufficient to promote a mental representation of exact number, but that the crucial factor would be the existence of a counting routine - which the Mundurukú do not have.

Even stronger evidence for the role of counting for the development of exact numerical representations comes from observations of deaf adults living in a numerate society, leading to the same conclusions as the two studies described above. The deaf individuals investigated by Spaepen and colleagues (2010) live in Nicaragua. Despite having no language model at hand, they have access to other aspects of culture which may foster the development of number concepts, for example, observing other people's hand gestures. These deaf adults have developed their own gestures for communication, called homesigns, which they also use to communicate about number. The four observed adult men showed no congenital cognitive deficits, performed as well as hearing siblings and friends on mental rotation tasks, held jobs, made money, and interacted socially with hearing friends and relatives. However, the homesigners did not consistently extend the correct number of finger for set sizes larger than three. Furthermore, they did not always correctly match the number of items in one set to a target set containing more than three items. Overall, the homesigners failed to appreciate the one-to-one correspondence guaranteeing numerical equivalence. The authors concluded that individuals who lack input from a conventional language structure, do not spontaneously develop representations of exact numerosities above the subitizing range (three or four items, which can be individualized in parallel due to the object tracking system). They speculated that although the homesigners at least partially mastered the monetary system (identifying currency and rating relative values), their numerical homesigns are not embedded in accounting routine. Thus, the homesigners lacked summary symbols for each integer 
representing the cardinal value and the principle of the successor function (that each natural number $n$ has a successor that is exactly $n+1$ ).

However, the question remains what happens if adult humans without a counting routine, without number words for numerosities beyond five and thus without concepts for exact quantities larger than that are exposed to a more elaborate number word system. If language really is the constituting factor for exact numerical representations, experience with a counting system should be sufficient for that even in adults.

Dehaene and colleagues (2008) tested 33 Mundurukú children and adults of varying exposure to formal education and thus with varied exposure to the Portuguese number word system. Using number line tasks in different modalities (sets of dots, sequences of tones, Mundurukú number word [composites], and Portuguese number words for bilinguals), they could show that mere exposure to the Portuguese counting system was not enough to evoke an exact (linear) magnitude representation on a number line task. Only the individuals with the highest number of years of formal education - who had experience with addition and subtraction procedures - were able to provide with exact responses for Portuguese number words. The authors concluded that experience with arithmetic and measurement yields the intuition that all consecutive numbers are separated by the same interval +1 . However, even the most educated Mundurukú participants of this study did not extend this principle to the Mundurukú number words, speculatively because this cultural device "does not emphasize measurement or invariance by addition and subtraction as defining features of number, contrary to Western numerical systems" (Deheane et al., 2008: 1219).

So far, it is safe to conclude that there is a very strong argument that the development of exact numerical representations is dependent on language. For the smallest number range, developmental studies imply a crucial supporting role of morphological number markers (most importantly, plural markers). For numbers beyond the subitizing range, a counting routine seems to be necessary to develop a concept of the successor function of numbers, which in turn allows for exact number representations. However, the presented evidence from bilingual Mundurukú speakers implies that this may not be the whole story and that experience with adding and subtracting could play an important role as well. Hence, the next section will explore the relation between language and arithmetic. 


\section{Arithmetic fact retrieval in first and second language}

Since the 1990s more and more scientific evidence points to the languagesensitivity - if not language dependency - of arithmetic fact retrieval (Bernardo, 2001). Interestingly, one of the first experimental studies showed that a firstlanguage advantage in bilinguals is usually only found for arithmetic (response times and accuracy), but not for the manipulation of number words in general (Frenck-Mestre \& Vaid, 1993). Arithmetic facts were usually highly overlearned and automatized only in the first language (L1) and showed weaker associations between problems and answers in the second language (L2).

\subsection{Language-sensitivity of arithmetic fact retrieval depends on language of instruction}

However, it is important to note that this language-sensitivity is not constituted by an advantage of L1 in general, but that the crucial factor is the language of first arithmetic instruction and/or the language of training (Bernardo, 2001; see also Saalbach et al., 2013). Bernardo (2001) noted that this effect is not consistent with abstract, format-independent number fact representations. However, it fits into the encoding complex model by Campbell and Clark (1988). Saalbach and colleagues (2013) pointed out that it is also in line with the triple-code model by Dehaene and Cohen (1997), which assumes an abstract, format-independent representation of numerical quantity, but not of arithmetic operations.

\subsection{Language effects occur in trained exact, but not in trained approximate, arithmetic operations}

Spelke and Tsivkin (2001) observed the same training-language effects for arithmetic operation in adult bilinguals, but added an important new specification. Notably, they found language switching costs (slower responses for problems presented in the untrained language) for trained exact arithmetic operations (like repeatedly adding 54 or 63 to a given number, addition in base 6 or base 8), but not for trained approximate arithmetic operations (like approximation of cube roots, approximation of logarithmic bases). They proposed that natural language contributes to the representation of large, exact numbers but not to our biologically inherited approximate number representations and offered a possible explanation 
for this special role of language within the domain of numerical representations. The authors argued that language was the first evolutionary developed representational system allowing for the combination of input from different modalities (e.g. spatial layouts and smell). They further elaborated that the two known biological (and thus language-independent) core systems for exact number representations, namely, an approximate system for large numerosities and a small number system (see above), both fail to represent all the complementary aspects of number. The "large approximate system fails to represent each member of a set as a persisting individual," whereas the "small number system fails to represent a group of individuals explicitly as a set” (Spelke \& Tsivkin, 2001: 82). They suggested that the natural counting system may allow humans to combine these two distinct types of representation into a representation of sets of individuals whose cardinality increases as new individuals are added to the set. As language was necessary to link the two limited systems, the new hybrid system - which captures the benefits and overcomes the limits of each language-independent system - depends on language.

In summary, Spelke and Tsivkin (2001) suggest that the symbolic nature of the verbal counting system brings together the distinct features of small exact numerosity processing and large number approximation. This theory provides an explanation for all of the phenomena described so far in this chapter. However, at least for the context of bilingual or multilingual instruction, the question remains what happens if individuals have been overlearning arithmetic facts in a second language for years.

\subsection{Developmental change of language effects for arithmetic fact retrieval}

Van Rinsveld et al. (2015) investigated this phenomenon in Luxembourg, where the German-speaking individuals usually attend the first six years of primary school with instruction in their mother tongue, but then switch to Frenchlanguage secondary education. Implementing a cross-sectional study design using five different age groups from the seventh grade to adulthood, they could show that simple addition fact retrieval was almost equally accurate after extended practice in French, but still faster in German. For addition including a carry procedure, even the young adults were faster and more accurate in German, although the performance differences between the two languages diminished somewhat.

So in the case of highly efficient bilinguals who can solve simple arithmetic facts almost equally well in two languages, the question remains whether they 
will build new associations within the numerical fact networks in the second language, or become more and more efficient in translating from one language into the other (Lin et al., 2012; Wang et al., 2007), or will use a different strategy altogether?

\subsection{Which strategy is used for arithmetic fact retrieval in L2?}

The possible relations between arithmetic fact representations in different languages and strategies used for fact retrieval in L2 have been investigated using functional magnetic resonance imaging (fMRI) studies (Van Rinsveld et al., 2017) and EEG studies using event-related potentials (ERP: e.g., Salillas \& Wicha, 2012). fMRI studies measure differences in oxygenated and de-oxygenated blood flow in the brain and thus allow for three-dimensional localizations of active regions in the functioning human brain with high spatial, but low temporal resolution (Windhorst \& Johannson, 2013). Event-related potentials use EEG to measure electrical brain activity following specific "events" and have a very high temporal, but low spatial, resolution (Windhorst \& Johannson, 2013).

These studies foster the conclusion that arithmetic in L2 is mediated by the Arabic digit code and not the verbal code of L1 (as expected if participants would translate the problems into L1). Specifically, van Rinsveld and colleagues (2017: 27) asked participants to solve visually and auditively presented arithmetic problems in L1 and L2 in the scanner and observed additional brain activation in the auditive task in the L2 condition compared to the other conditions in occipito-temporal areas (known to underlie the processing of Arabic digits) and in the precuneus area (important for visual imagery). They concluded that arithmetic in L2 may be performed "with the help of a mental visual support, such as via imagining the heard numbers in their visual symbolic form."

Summing up, both the development of large, exact number concepts and the mental manipulation of exact quantities (arithmetic) seem to be languagedependent. Furthermore, diminishing the costs of mental arithmetic in L2 (or more specifically in a language different from the one used in first mathematical instruction at school) appears to rely on switching to another representational format altogether, namely, to the visual symbolic code of Arabic digits. As the next section will show, this might generate even more problems for bilingual learners who already have mathematical difficulties. 


\subsection{The role of symbolic number processing for arithmetic}

In 2007, Rouselle, and Noël presented a theory claiming that a core deficit in children with developmental dyscalculia may not lie in their approximate number system and thus in processing numerosity per se, but in their ability to access number magnitude from symbols. This access deficit hypothesis has triggered extensive research tapping the differential roles of symbolic and non-symbolic numerical magnitude processing skills. A review of these studies concluded that there is indeed consistent and robust evidence across studies and different age groups for a correlation between weak performance in symbolic numerical tasks (using Arabic digits) and low math achievement, whereas conflicting findings have been reported for respective non-symbolic formats (De Smedt et al., 2013). This stronger relation between math achievement and symbolic vs. non-symbolic number processing means that children with mathematical difficulties will most likely be less efficient in accessing the meaning of Arabic digits - namely their exact numerical value - compared to their peers. An even more pronounced problem will hence arise if they have to handle arithmetic tasks in multiple languages. This situation may be relevant for bilingual children whose caregivers cannot provide them with help in mathematics in the instructional language, or for children whose instructional language in mathematics changes during their educational years. As pointed out above, the most efficient strategy in such situations seems to be mentally switching to the Arabic code rather than translating problems into L1 - which, however, may not be possible to the same extent for children with difficulties in mathematics.

The question is then: Could children with problems in accessing the exact quantity of Arabic digits be provided with another set of symbols to bypass language-related difficulties in dealing with numbers?

\section{Finger representations as an alternative set of symbols}

During the last decade, neurocognitive research produced growing evidence that finger counting results in finger-based numerical representations in the sense of embodied cognition (Fischer \& Brugger, 2011) with a sub-base 5 (Domahs et al., 2008) which can still be found in healthy adults (Domahs et al., 2010). Several studies also showed that finger gnosis, the ability to differentiate between the fingers (Gerstmann, 1940), predicts calculation abilities even if general cognitive and motor abilities are controlled for (see Berteletti \& Booth, 2015 for a review). 
The reasons for these connections between finger representations and number representations are still unclear. Some authors suggest that there are anatomically neighboring brain areas for finger- and number processing; others also suggest a functional connection (see Berteletti \& Booth, 2015 for an overview of this discussion). Finger-based numerical representations can be seen as a prototypical example of embodied cognition, hence the question arises which aspect of finger counting may be crucial for this: the one-to-one correspondence of fingers and cardinal value, the additional somatosensory route of perception, or the automatic processing of canonical finger patterns (Brissiaud, 1992).

Priming studies in adults showed that canonical finger patterns for showing numbers (e.g., all 5 fingers of one hand and 2 fingers of the other hand to show 7) were processed as exact magnitudes like Arabic numbers, whereas non-canonical finger patterns (e.g., 3 fingers of one hand and 4 fingers of the other hand to show 7) were processed as approximate magnitudes like dot patterns (Di Luca, Lefevre \& Pesenti, 2010; Di Luca \& Pesenti, 2008). This finding means that canonical finger patterns may have the same symbolic character as Arabic digits, providing a possible alternative route to exact numerical quantity representations. This interpretation was confirmed by Krinzinger et al. (2011) in a developmental fMRI study. Berteletti and Booth (2015: 8) also concluded that educational practices should encourage the use of fingers "as a functional link between numerical quantities and their symbolic representation." Another advantage of this may well be that fingers provide an external support for arithmetic problems, decreasing the working memory load and therefore increasing the efficiency of mental calculation (Berteletti \& Booth, 2015).

\section{Summary and discussion}

In summary, several different effects of language on the processing of exact number can hardly be explained by a theory of cultural universality (Au, 1983).

First, the number of grammatical plural markers used in a language influences the speed with which children acquire the exact number concepts of one, two, and three (see Section 1.4). Second, adult humans who speak a language lacking words for exact numbers higher than three or four do not present with exact number concepts even in nonverbal tasks (see Section 2). Both lines of evidence defy the view of the language-irrelevant hypothesis, but rather support the claim that the acquisition of concepts of large, exact numerosities (or cardinality) is language-driven and relies on learning respective linguistic representations (or symbols). 
Third, the mental manipulation of exact numbers (or arithmetic) is also language-dependent, as arithmetic fact retrieval is usually faster and more accurate in the language of instruction (see Section 3). Interestingly, more efficient arithmetic fact retrieval in a secondary language seems to correspond to a stronger reliance on the visual imagery of Arabic digits and thus to a change in the modality of mental representations rather than on faster translational processes or building a new mnemonic network of arithmetic facts altogether. As the core deficit of children with low math achievement seems to lie in symbolic, exact number processing rather than in non-symbolic, approximate number processing (see Section 3.5), it is safe to assume that doing mental arithmetic in a secondary language should pose even larger difficulties for these children compared to their typically developing peers.

A solution to this problem may lie in the explicit use of canonical finger configurations to depict exact number in formal and informal educational settings, as they seem to possess the same symbolic characteristics as Arabic digits and may thus provide children with an additional developmental route to exact number representations (see Section 4). In conclusion, the automatized processing of canonical finger patterns might enhance symbolic (and therefore exact) processing of numerical magnitudes especially in children presenting with languagerelated difficulties in mathematics or exhibiting less efficient processing of Arabic digits.

\section{References}

Au, Terry Kit-Fong (1983): Chinese and English counterfactuals: The Sapir-Whorf hypothesis revisited. Cognition 15, 155-187.

Barner, David, Thalwitz, Dora, Wood, Justin, Yang, Shu-Ju \& Carey, Susan (2007): On the relation between the acquisition of singular-plural morpho-syntax and the conceptual distinction between one and more than one. Developmental Science 10, 365-373.

Bernardo, Ana B. (2001): Asymmetric activation of number codes in bilinguals: Further evidence for the encoding complex model of number processing. Memory \& Cognition 29 (7), 968-976.

Berteletti, Ilaria \& Booth, James R. (2015): Perceiving fingers in single-digit arithmetic problems. Frontiers in Psychology 6, 226. doi:10.3389/fpsyg.2015.00226.

Brannon, Elizabeth M. \& Van de Walle, Gretchen A. (2001): The development of ordinal numerical competence in young children. Cognitive Psychology 43, 53-81.

Brissiaud, Remi (1992): A toll for number construction: Finger symbol sets. In Bideaud, J., Meljac, C., Fischer, J. P. (Eds.): Pathways to Number: Children's Developing Numerical Abilities. Hillsdale, NJ: Lawrence Erlbaum 41-65. 
Butterworth, Brian (2010): Foundational numerical capacities and the origins of dyscalculia. Trends in Cognitive Sciences 14, 534-541.

Campbell, Jamie I.D. \& Clark, James M. (1988): An encoding-complex view of cognitive number processing: Comment on McCloskey, Sokol, andGoodman (1986). Journal of Experimental Psychology: General 117, 204-214.

Carey, Susan (2001): Cognitive foundations of arithmetic: Evolution and ontogenesis. Mind \& Language 16, 37-55.

Condry, Kirsten F. \& Spelke, Elizabeth S. (2008): The development of language and abstract concepts: The case of natural numbers. Journal of Experimental Psychology: General 137, $22-38$.

De Smedt, Bert, Noël, Marie-Pascale, Gilmore, Camilla \& Ansari, Daniel (2013): How do symbolic and non-symbolic numerical magnitude processing skills relate to individual differences in children's mathematical skills? A review of evidence from brain and behaviour. Trends in Neuroscience and Education 2 (2), 48-55.

Dehaene, Stanislas (1997): The Number Sense: How the Mind Creates Mathematics. New York, NY: Oxford University Press.

Dehaene, Stanislas \& Cohen, Laurent (1997): Cerebral pathways for calculation: Double dissociation between rote verbal and quantitative knowledge of arithmetic. Cortex 33 (2), 219-250. doi:doi.org/10.1016/S0010-9452(08)70002-9.

Dehaene, Stanislas, Izard, Veronique, Spelke, Elizabeth \& Pica, Pierre (2008): Log or linear? Distinct intuitions of the number scale in western and amazonian indigene cultures. Science 320, 1217. doi:10.1126/science.1156540.

Di Luca, S., Lefèvre, N. \& Pesenti, M. (2010): Place and summation coding for canonical and non-canonical finger numeral representations. Cognition 117, 95-100.

Di Luca, Samuel \& Pesenti, Mauro (2008): Masked priming effect with canonical finger numeral configurations. Experimental Brain Research 185, 27-39.

Domahs, Frank, Krinzinger, Helga \& Willmes, Klaus (2008): Mind the gap between both hands: Evidence for internal finger-based number representations in children's mental calculation. Cortex 44, 359-367.

Domahs, Frank, Moeller, Korbinian, Huber, Stefan, Willmes, Klaus \& Nuerk, Hans-Christoph (2010): Embodied numerosity: Implicit hand-based representations influence symbolic number processing across cultures. Cognition 116, 251-266.

Fischer, Martin H. \& Brugger, Peter (2011): When digits help digits: Spatial-numerical associations point to finger counting as prime example of embodied cognition. Frontiers in Psychology 2 (260). doi:10.3389/fpsyg.2011.00260.

Franzon, Francesca, Zanini, Chiara \& Rugani, Rosa (2019): Do non-verbal number systems shape grammar? Numerical cognition and number morphology compared. Mind \& Language 34, 37-58.

Frenck-Mestre, Cheryl \& Vaid, Jyotsna (1993): Activation of number facts in bilinguals. Memory \& Cognition 21 (6), 809-818.

Gelman, Rochel \& Gallistel, Charles R. (1978): The Child's Understanding of Number. Cambridge, Mass.: Harvard University Press.

Gerstmann, Josef (1940): Syndrome of finger agnosia, disorientation for right and left, agraphia and acalculia. Archives of Neurology and Psychiatry 44, 398-408.

Gordon, Peter (2004): Numerical cognition without words: Evidence from Amazonia. Science 306, 496-499. 
Greeno, James G., Riley, Mary S. \& Gelman, Rochel (1984): Conceptual competence and children's counting. Cognitive Psychology 16, 94-143.

Hoijer, Harry (1971): The Sapir-Whorf-hypothesis. In Hoyer, Harry (ed.): Language in Culture. Conference on the Interrelations of Language and Other Aspects of Culture. Seventh Impression. Chicago: Chicago University Press, 1971, 92-105.

Koudier, Sid, Halberda, Justin, Wood, Justin \& Carey, Susan (2006): Acquisition of English number marking: The singular-plural distinction. Language Learning and Development 2, 1-25.

Krinzinger, Helga, Koten, Jan Willem, Horoufchin, Houpand, Kohn, Nils, Arndt6, Dominique, Sahr, Katleen, Konrad1, Kerstin \& Willmes, Klaus (2011): The role of finger representations and saccades for number processing: An fMRI study in children. Frontiers in Psychology. doi:10.3389/fpsyg.2011.00373.

Le Corre, Mathieu, Van de Walle, Gretchen, Brannon, Elizabeth M. \& Carey, Susan (2006): Re-visiting the competence/performance debate in the acquisition of the counting principles. Cognitive Psychology 52, 130-169.

Lin, J.-F.L., Imada, T. \& Kuhl, P.K. (2012): Mental addition in bilinguals: An fMRI study of task-related and performance-related activation. Cerebral Cortex 22 (8), 1851-1861.

Pica, Pierre, Lemer, Cathy, Izard, Véronique \& Dehaene, Stanislas (2004): Exact and approximate arithmetic in an amazonian indigene group. Science 306, 499-503.

Rousselle, Laurence \& Noël, Marie-Pascale (2007): Basic numerical skills in children with mathematics learning disabilities: A comparison of symbolic vs non-symbolic number magnitude processing. Cognition 102 (3), 361-395.

Rousselle, Laurence, Palmers, Emmanuelle \& Noël, Marie-Pascale (2004): Magnitude comparison in preschoolers: What counts? Influence of perceptual variables. Journal of Experimental Child Psychology 87, 57-84.

Saalbach, Henrik, Eckstein, Doris, Andri, Nicoletta, Hobi, Reto \& Grabner, Roland H. (2013): When language of instruction and language of application differ: Cognitive costs of bilingual mathematics learning. Learning and Instruction 26, 36-44.

Salillas, Elena, \& Wicha, Nicole Y. (2012): Early learning shapes the memory networks for arithmetic: Evidence from brain potentials in bilinguals. Psychological Science 23(7), 745-55.

Sapir, Edward (1949): In Mandelbaum, D. (Ed.): Selected Writings of Edward Sapir. Berkeley, CA: University of California Press. 1-617

Sarnecka, Barbara W. \& Carey, Susan (2008): How counting represents number: What children must learn and when they learn it. Cognition 108, 662-674.

Sarnecka, Barbara W. \& Gelman, Susan A. (2004): Six does not just mean a lot: Preschoolers see number words as specific. Cognition 92, 329-352.

Sarnecka, Barbara W., Kamenskaya, Valentina G., Yamana, Yuko, Ogura, Tamiko \& Yudovina, Yulia B. (2007): From grammatical number to exact numbers: Early meanings of 'one', 'two', and 'three' in English, Russian, and Japanese. Cognitive Psychology 55, 136-168.

Spaepen, Elizabet, Coppola, Marie, Spelke, Elizabeth S., Carey, Susan E. \& Goldin-Meadow, Susan (2010). Number without a language model. Proceedings of the National Academy of Sciences of the United States of America 108, 3163-3168.

Spelke, Elizabeth.S. \& Tsivkin, Sanna (2001): Language and number: A bilingual training study. Cognition 78, 45-88.

Van Rinsveld, Amandine, Brunner, Martin, Landerl, Karin, Schiltz, Christine \& Ugen, Sonja (2015): The relation between language and arithmetic in bilinguals: Insights from 
different stages of language acquisition. Frontiers in Psychology 6 (265). doi:10.3389/ fpsyg.2015.00265.

Van Rinsveld, Amandine, Dricot, Laurence, Guillaume, Mathieu, Rossion, Bruno \& Schiltz, Christine (2017): Mental arithmetic in the bilingual brain: Language matters. Neuropsychologia 101, 17-29.

Wang, Yue, Lin, Lotus, Kuhl, Patricia \& Hirsch, Joy (2007): Mathematical and linguistic processing differs between native and second languages: An fMRI study. Brain Imaging Behaviour 1 (3-4), 68-82.

Whorf, Benjamin Lee (1956): In Carroll, John B. (Ed.): Language, Thought, and Reality: Selected Writings of Benjamin Lee Whorf. Cambridge, MA: MIT Press. 1-294

Windhorst, Uwe \& Johannson, Hakan (2013): Modern Techniques in Neuroscience Research. Berlin, Heidelberg: Springer.

Wynn, Karen (1990): Children's understanding of counting. Cognition 36, 155-193.

Wynn, Karen (1992): Children's acquisition of the number words and the counting system. Cognitive Psychology 24, 220-251. 